\title{
Intravenous and intraocular ganciclovir for CMV retinitis in patients with AIDS or chemotherapeutic immunosuppression
}

\author{
GEORGE L DAIKOS, JOSE PULIDO, SHASHI B KATHPALIA, \\ AND GEORGE GEE JACKSON
}

From the Section of Infectious Diseases, Department of Medicine, and the Department of Ophthalmology, University of Illinois College of Medicine, Chicago, Illinois, USA

SUMMARY The efficacy and toxicity of ganciclovir given by intravenous or intravenous plus intravitreal injection were studied in nine patients with cytomegalovirus (CMV) retinitis; seven with AIDS and two with drug induced immunodeficiency. Five patients had retinitis with macular involvement in six sighted eyes; six patients had only peripheral retinitis in seven eyes. In two patients (two eyes) with macular involvement intravenous plus intravitreal injection of ganciclovir preserved sight; intravenous infusion alone did not in four eyes of three other patients. In seven eyes (six patients) with peripheral retinitis vision was retained regardless of the route of ganciclovir treatment. Following intravenous ganciclovir drug levels in the vitreous fluid were $1 \cdot 4-2 \cdot 2 \mathrm{mmol} / \mathrm{l}$, that is, 44 and $65 \%$ of the concomitant serum concentration. Clinically and at necropsy three eyes showed no evidence of toxicity from intravitreal injection of ganciclovir. All of five patients with AIDS who received intravenous ganciclovir for more than one week developed leucopenia. CMV retinitis of the macula may be benefited with minimal drug toxicity by intravitreal injection of ganciclovir. Treatment of peripheral CMV retinitis in patients with AIDS may be optional.

Necrotising retinitis caused by cytomegalovirus (CMV) is one of the serious manifestations of immune deficiency states. ${ }^{12}$ It has the potential for producing blindness and seriously depreciating the quality of life. In patients immunosuppressed by medication CMV retinitis can be controlled by withdrawal of the immunosuppressive drugs. In patients with the acquired immune deficiency syndrome (AIDS) the irreversibility of the immune suppression makes progression of CMV retinitis the expected course. The increasing prevalence of AIDS and of chemotherapy causing immunosuppression has increased the occurrence of CMV retinitis and emphasised the need for effective treatment. ${ }^{34}$

The most effective antiviral drug for the treatment of CMV infections is 9-(1,3 dihydroxy 2propoxymethyl) guanine (ganciclovir). ${ }^{5-11}$ Its use

Correspondence to George Gee Jackson, MD, University of Illinois College of Medicine, 840 South Wood Street, Chicago, Illinois 60612, USA. offers the potential for stabilising and reversing CMV retinitis; however, it does not cure the disease. The requirement for long-term intravenous therapy often produces haematopoietic suppression. Treatment to prevent blindness has shown some benefit by funduscopy and visual acuity as measures of the effect. In the absence of controlled comparative data a continuing critical appraisal of experience with ganciclovir treatment is necessary to judge its benefit, pharmacological requirements, toxicity, and the therapeutic options.

\section{Material and methods}

The diagnosis of CMV retinitis was based on the characteristic findings on funduscopic examination, absence of another explanation of the retinitis, and cultures for CMV. Cultures of peripheral blood leucocytes, throat secretions, and urine were made before treatment, and at approximately monthly 
intervals when prolonged treatment was given. The investigational protocol was approved by the Institutional Review Board for research involving humans, and informed consent was obtained from the patients. Patients were admitted to hospital for two weeks' initial treatment. Ganciclovir $2.5 \mathrm{mg} / \mathrm{kg}$ was given intravenously every 8 hours (except that one patient received $5 \mathrm{mg} / \mathrm{kg}$ ). If the dose was tolerated without haemosuppression, a single daily dose was continued for 14-20 days. If the leucocyte count decreased below $1 \times 10^{\%} / 1$ or by more than $50 \%$ of the initial count, further doses were deferred until reversal of the condition, and then ganciclovir was started again. After the initial course the treatment was continued, usually at home, with doses of $2.5 \mathrm{mg} / \mathrm{kg}$ daily on five days of the week.

Ophthalmic examinations were performed by the same ophthalmologist (JP) at least twice weekly before and during the initial treatment and frequently thereafter. The visual acuity was graded by the Snellen test and by standard terms for gross impairment: ability to count fingers, discern hand motion, and perceive light. Photographs of the retina were taken before and during treatment to record perceived changes. The response to therapy was rated as improvement, stabilisation, or progression.

In three patients blood samples were drawn at frequent intervals following infusion of $5 \mathrm{mg} / \mathrm{kg}$ or 2.5 $\mathrm{mg} / \mathrm{kg}$ for measurements of the plasma drug level. Instillation of intravitreal ganciclovir was done in the outpatient operating room by transcleral injection under direct visualisation. After removal of $0 \cdot 1-0 \cdot 2$ $\mathrm{ml}$ of vitreous fluid, $200-400 \mu \mathrm{g}$ of ganciclovir in $0 \cdot 1$ $\mathrm{ml}$ sterile water was injected. The procedure was performed two hours after the completion of the intravenous infusion of a dose of ganciclovir, and a simultaneous sample of serum was obtained.

\section{Results}

Of nine patients with necrotising CMV retinitis seven had AIDS and two were immunosuppressed for maintenance of a renal homograft. All of them had bilateral CMV retinitis. CMV was isolated from at least one clinical specimen from all but two patients. For the evaluation of treatment the patients were categorised according to macular involvement or only peripheral retinitis. The data are presented in Table 1.

\section{MACULAR RETINITIS}

Of five patients with necrotising retinitis involving the macula three were treated with intravenous ganciclovir and became totally or nearly totally blind during or shortly after treatment. One patient received the drug for only two days and withdrew because of fear of drug toxicity. He became totally blind within a few weeks. Another patient progressed to total blindness during the first week of treatment, which was then discontinued. A third patient had small areas of retinitis involving the macula bilaterally. Initially the visual acuity was good, 20/20, but on a regular schedule of treatment with intravenous ganciclovir $(2.5 \mathrm{mg} / \mathrm{kg}$ five times a week) over a period of 43 days until death vision deteriorated progressively to $20 / 70$ (OS) and to perceiving hand motion (OD).

In two eyes of two patients with macular retinitis ganciclovir was given by intraocular injection as well as by intravenous infusion. Following the intravitreal injection the retinal lesions improved in each of the eyes as judged by funduscopy and by visual acuity. One patient's visual acuity of counting fingers at 5 feet (CF 5) $(1.5 \mathrm{~m})$ was improved to $20 / 40$ and retained for one month after the intravitreal injection until death occurred from other causes. The other patient's visual acuity deteriorated from $20 / 100$ to CF 5 with intravenous therapy alone, but was improved to 20/200 and retained for approximately two months after the intraocular injection of ganciclovir; in the third month vision deteriorated while the patient was receiving intravenous doses of ganciclovir intermittently owing to toxicity.

\section{PERIPHERA L RETINITIS}

In seven eyes of six patients CMV retinitis involved only the peripheral retina. In all of them visual acuity was good (20/20 and 20/40), and it remained so until the time of death regardless of whether the treatment regimen included intraocular treatment or only intravenous therapy. Four patients with AIDS died within one to six months. In two patients with renal allografts ganciclovir was given only intravenously and the dose of immunosuppressive drugs reduced. The retinitis healed and remained quiescent without further treatment for eight months and one month respectively.

PHARMACOLOGY AND TOXICOLOGY

Intravenous infusion of $2.5 \mathrm{mg} / \mathrm{kg}$ of ganciclovir produced peak serum levels of 11 to $19.4 \mathrm{mmol} / \mathrm{l} ; 5$ $\mathrm{mg} / \mathrm{kg}$ in one patient gave a peak level of $25.6 \mathrm{mmol} / \mathrm{l}$. Trough levels after eight hours were $1 \mathrm{mmol} / \mathrm{l}$ or less. Two hours after intravenous infusion ganciclovir levels in the vitreous fluid were approximately onehalf the concentration in serum. Concomitant serum and vitreous fluid concentrations of ganciclovir in two eyes of two patients are given in Table 2. Based on a distribution volume of $11.7 \mathrm{ml}^{11}$ the calculated drug levels after intraocular injections of 200,300, and 400 $\mu \mathrm{g}$ of ganciclovir were initially approximately 70 , 105 , and $140 \mathrm{mmol} / \mathrm{l}$ respectively. One month after 
Table 1 Necrotising CMV retinitis treated with ganciclovir

\begin{tabular}{|c|c|c|c|c|c|c|c|c|c|}
\hline \multirow{3}{*}{$\begin{array}{l}\text { Patient data } \\
\text { Treatment: }\end{array}$} & \multicolumn{5}{|c|}{ Patients with macular involvement } & \multicolumn{4}{|c|}{ Patients with peripheral disease } \\
\hline & $l$ & 2 & 3 & 4 & 5 & 6 & 7 & 8 & 9 \\
\hline & \multicolumn{3}{|c|}{ Intravenous only } & \multicolumn{2}{|l|}{ Intraocular } & \multicolumn{4}{|c|}{ Intravenous only } \\
\hline Age, yr & 30 & 42 & 30 & 28 & 44 & 28 & 30 & 42 & 44 \\
\hline Sex & Male & Male & Male & Male & Male & Female & Male & Female & Female \\
\hline IV dose (g) & $0 \cdot 8$ & $5 \cdot 4$ & $9 \cdot 2$ & $8 \cdot 9$ & $9 \cdot 9$ & $5 \cdot 4$ & $3 \cdot 6$ & $5 \cdot 8$ & $5 \cdot 5$ \\
\hline Duration, days & 2 & 6 & 53 & 90 & 30 & 15 & 8 & 14 & 20 \\
\hline \multicolumn{10}{|l|}{ Retinal involvement ${ }^{a}$} \\
\hline \multicolumn{10}{|l|}{ Before treatment } \\
\hline OS & $\mathbf{M}$ & Old & $\mathbf{M}$ & $\mathrm{P}$ & M & $\mathbf{P}$ & $\mathbf{P}$ & Old & $\mathbf{P}$ \\
\hline OD & $\mathbf{M}$ & $\mathbf{M}$ & $\mathbf{M}$ & $\mathbf{M}$ & $\mathbf{P}$ & ON & $\mathbf{P}$ & $\mathbf{P}$ & Old \\
\hline \multicolumn{10}{|l|}{ After treatment } \\
\hline OS & $\mathrm{NC}$ & $\mathrm{NC}$ & $\downarrow$ & $\mathrm{NC}^{*}$ & $\uparrow^{*}$ & $\downarrow$ & $\uparrow$ & NC & $\uparrow$ \\
\hline OD & $\mathrm{NC}$ & $\downarrow$ & $\downarrow$ & $\downarrow / \uparrow *$ & $\uparrow$ & $\downarrow$ & $\uparrow$ & $\uparrow$ & NC \\
\hline \multicolumn{10}{|l|}{ Visual acuity $^{\mathrm{b}}$} \\
\hline \multicolumn{10}{|l|}{ Before treatment } \\
\hline OS & NLP & NLP & $20 / 20$ & $20 / 20$ & CF5 & $20 / 20$ & $20 / 30$ & CF5 & $20 / 20$ \\
\hline OD & $20 / 200$ & CF5 & $20 / 20$ & $20 / 100$ & $20 / 20$ & NLP & $20 / 40$ & $20 / 20$ & CF5 \\
\hline \multicolumn{10}{|l|}{ After treatment } \\
\hline OS & NLP & NLP & $20 / 70$ & $20 / 20^{*} \quad 2$ & $20 / 40^{*}$ & $20 / 20$ & $20 / 30$ & CF5 & $20 / 20$ \\
\hline OD & $20 / 200$ & NLP & $\mathrm{HM}$ & CF5 20/200*2 & $20 / 20$ & NLP & $20 / 40$ & $20 / 20$ & CF5 \\
\hline \multicolumn{10}{|l|}{ Viral cultures } \\
\hline \multicolumn{10}{|l|}{ Before treatment } \\
\hline Blood & NA & + & - & - & - & - & - & - & - \\
\hline Urine & NA & - & + & + & + & - & + & + & - \\
\hline Throat & NA & + & - & + & + & + & + & - & - \\
\hline \multicolumn{10}{|l|}{ After treatment } \\
\hline Blood & NA & - & + & - & - & - & - & - & - \\
\hline Urine & NA & - & + & - & - & - & + & - & - \\
\hline Throat & NA & NA & + & - & - & - & - & - & - \\
\hline \multicolumn{10}{|l|}{ Leucopenia } \\
\hline$<1 \times 10^{9} / \mathrm{l} />50 \%$ fall & No & No & Yes & Yes & Yes & Yes & Yes & No & No \\
\hline
\end{tabular}

${ }^{\mathrm{a}} \mathrm{M}=$ macular lesion, $\mathrm{P}=$ peripheral retina, $\mathrm{ON}=$ optic nerve, ${ }^{*}=200-400 \mu \mathrm{g}$ intraocularly, $\mathrm{NC}=$ no change,$\downarrow=$ progression of lesion(s),

$\uparrow=$ improvement of lesion(s). ${ }^{\text {N }} \mathrm{NLP}=$ no light perception, $\mathrm{CF} 5=$ count fingers at $5 \mathrm{feet}(1 \cdot 5 \mathrm{~m}), \mathrm{HM}=$ perceive hand $\mathrm{motion}$.

${ }^{\mathrm{N} A}=$ not available.

intraocular injection and continuing intravenous doses, the inoculated eye of one patient had a slightly higher concentration of drug than the opposite eye.

In one patient CMV viraemia in the leucocyte buffy coat persisted, though peak ganciclovir serum levels of $19.4 \mathrm{uM} / \mathrm{ml}$ were attained. In all other patients viral cultures for CMV were rarely positive during the period when ganciclovir was given.

Of seven patients with AIDS and an initial leucocyte count of $>1 \times 10^{9} / 1$ five received ganciclovir for more than one week and all of them developed leucopenia. In four the granulocyte count decreased to less than $1 \times 10^{9} / 1$ and in the other decreased more than $50 \%$. All seven patients with AIDS died. One patient had reactivated Pneumocystis carinii pneumonia and also CMV pneumonia, another had CMV pneumonia with probable disseminated CMV; two other patients had overtly disseminated CMV infection.

CMV retinitis was confirmed in six eyes enucleated from three patients at necropsy, including three injected with ganciclovir. Virus was recovered from the retina of one eye. In none of the patients could another infection of the retina be identified histologically or by cultures for viruses and bacteria. The three eyes that were injected with ganciclovir showed no gross or microscopic evidence of drug toxicity.

\section{Discussion}

With a disease as potentially devastating but as variable as necrotising CMV retinitis, and a drug with the potential efficacy and toxicity of ganciclovir, controlled trials would be important but possibly unethical. The experience presented here, as in other reports, is limited and without controls on which to base firm conclusions. In the absence of such investigation it is important to develop criteria for selection of patients and expectations from treatment with ganciclovir. A fundamental question is whether all patients with necrotising CMV retinitis will benefit from ganciclovir treatment. In patients with AIDS, 
Table 2 Levels of ganciclovir in serum and vitreous fluid, $\mathrm{mmol} / \mathrm{l}$

\begin{tabular}{llllll}
\hline $\begin{array}{l}\text { Patient } \\
\text { no. }\end{array}$ & $\begin{array}{l}\text { Duration of } \\
\text { treatment } \\
2 \cdot 5 \mathrm{mg} / \mathrm{kg} \\
8 \text { hourly }\end{array}$ & $\begin{array}{l}\text { Timeafter } \\
\text { infusion }\end{array}$ & $\begin{array}{l}\text { Serum } \\
\text { (peak/trough) }\end{array}$ & Vitreous & $\begin{array}{l}\text { Ratio } \\
\text { V/S }\end{array}$ \\
& & & & \\
\hline 4 & Initial & $2 \mathrm{~h}^{*}$ & $3 \cdot 2$ & $1.4 \mathrm{OD}$ & $44 \%$ \\
5 & 4 days & $2 \mathrm{~h}^{*}$ & $3 \cdot 4(11 \cdot 0-1 \cdot 5)$ & $2 \cdot 2 \mathrm{OS}$ & $65 \%$ \\
& 30 days & $36 \mathrm{~h}$ & ND & 1.4 OS & \\
& & (necropsy) & & 0.9 OD & \\
\hline
\end{tabular}

$\mathrm{ND}=$ not done. ${ }^{*}$ Intravitreal injection.

cure of CMV retinitis with ganciclovir cannot be achieved. In its place the preservation of adequate vision might be a suitable aim. When CMV retinitis is only at a peripheral focus, the rate of progression to blindness may be slow and in patients with AIDS may be preceded by death. For those with initial involvement of the macula, intravenous treatment appears to be inadequate to save their sight. The anatomical differentiation of macular and non-macular involvement of the retina might serve as a basis for the categorisation of patients for different treatment regimens.

When the retinitis does not impair visual acuity, the avoidance of drug toxicity may be preferable to the expected gain when long-term intravenous ganciclovir is required. The levels of ganciclovir in vitreous fluid following intravenous administration of the recommended dose $(2.5 \mathrm{mg} / \mathrm{kg})$ may reach $50 \%$ of the concomitant serum concentration, but are probably inadequate for effective antiviral action against more than a third of the strains of CMV. ${ }^{5}$ Direct intravitreous injection of ganciclovir appears to be non-toxic and can produce inhibitory levels of drug at the site of infection for many hours. ${ }^{11-1+}$ When given in conjunction with intravenous therapy, the preservation of an inhibitory drug level in the eye may be present for a relatively long period. During that period CMV can be suppressed, the lesions stabilised, and vision preserved or improved. One patient has received multiple injections." The optimal and safe time intervals to repeat the intravitreal injections, if necessary to maintain the suppressive effect, are not known and may vary among patients. If acceptable, infrequent intravitreal injections could be programmed by clinical and funduscopic signs to fit the need.

The best regimen for maximum benefit and minimal drug toxicity remains unproved. Our experience suggests that ganciclovir therapy of peripheral retinal lesions can be individualised and observation without specific treatment justified. When macular lesions are initially present, intravitreal injection of ganciclovir may give the greatest likelihood of preserving sight with minimal drug toxicity.

All patients were treated under a compassionate plea protocol with the sponsorship of Burroughs Wellcome Company, Donna Cederberg, collaborating investigator. Appreciation is expressed to the following: Dr Greg Shipman, Chicago, IL. who referred three patients; Henry Balfour. MD, University of Minnesota, Minneapolis, Minnesota, and Paulo de Miranda, PhD, Burroughs Wellcome Company, Research Triangle Park, NC, who assayed the levels of ganciclovir in serum and vitreous; and Mary Rubenis for viral cultures done at the University of Illinois at Chicago. Core grant EYO1792 and training grant EYO7038 from the National Eye Institute, NIH, Bethesda, Maryland, helped support the work.

\section{References}

1 Pollard RB, Egbert PR, Gallagher JG, Merigan TC. Cytomegalovirus retinitis in immunosuppressed hosts. I. Natural history and effects of treatment with adenine arabinoside. Ann Intern Med 1980; 93: 655-64.

2 Egbert PR, Pollard RB, Gallagher JG, Merigan TC. Cytomegalovirus retinitis in immunosuppressed hosts. II. Ocular manifestations. Ann Intern Med 1980; 93: 664-70.

3 Palestine AG, Rodrigues MM, Macher AM, et al. Ophthalmic involvement in acquired immunodeficiency syndrome. Ophthalmology 1984; 91 : 1092-9.

4 Pepose JS, Holland GN, Nestor MS, Cochran AJ, Foos RY. AIDS pathogenic mechanisms of ocular disease. Ophthalmology 1985; 92: 472-84

5 Plotkin SA, Drew WL, Felsenstin D, Hirsch MS. Sensitivity of clinical isolates of human cytomegalovirus to 9-(1,3-dihydroxy-2propoxymethyl) guanine. J Infect Dis 1985; 152: 833-4.

6 Collaborative DHPG treatment study group. Treatment of serious cytomegalovirus infections with 9-(1,3-dihydroxy-2propoxymethyl) guanine in patients with AIDS and other immunodeficiencies. N Engl J Med 1986; 314: 801-5.

7 Palestine AG, Stevens G, Lane HC, et al. Treatment of cytomegalovirus retinitis with dihydroxy propoxymethyl guanine. Am J Ophthalmol 1986; 101: 95-101.

8 Felsenstein D, D'Amico DJ, Hirsch MS, et al. Treatment of cytomegalovirus retinitis with 9-[2-hydroxy-1(hydroxymethyl) ethoxymethyl] guanine. Ann Intern Med 1985; 103: 377-80.

9 Bach MC, Bagwell SP, Knapp NP, Davis KM, Hedsrom PS. 9(1,3-dihydroxy-2-propoxymethyl) guanine for cytomegalovirus infections in patients with the acquired immunodeficiency syndrome. Ann Intern Med 1985; 103: 381-2.

10 Holland GN, Sakamoto MJ, Hardy D, Sidikaro Y, Kreiger AE Frenkel LM, and the UCLA CMV Retinopathy Study Group. Treatment of cytomegalovirus retinopathy in patients with acquired immunodeficiency syndrome. Use of the experimental drug 9-[2-hydroxy-1-(hydroxymethyl) ethoxymethyl] guanine. Arch Ophthalmol 1986; 104: 1794-800.

11 Henry K, Cantrill H, Fletcher C, Chinnock BY, Balfour HH. Use of intravitreal ganciclovir (dihydroxy propoxymethyl guanine) for cytomegalovirus retinitis in a patient with AIDS. Am J Ophthalmol 1987; 103: 17-23.

12 Jabs DA, Wingard JR, DeBustros S, de Miranda P, Saral R, Santos GW. BWB759U for cytomegalovirus retinitis: intraocular drug penetration. Arch Ophthalmol 1986; 104: 1436-7.

13 Pulido J, Peyman GA, Lesar T, Vernot J. Intravitreal toxicity of hydroxyacyclovir (BW-B759U), a new antiviral agent. Arch Ophthalmol 1985; 103: 840-1.

14 Schulman J, Peyman GA, Horton MB, et al. Intraocular 9-([2hydroxy-l(hydroxymethyl) ethoxy] methyl) guanine levels after intravitreal and subconjunctival administration. Ophthalmic Surg 1986; 17: 429-32.

Accepted for publication 18 May 1987.

\section{然} (1) $\checkmark$

\section{8}

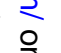
(2) . 